\title{
Observation and control in a model of a cell population affected by radiation
}

\author{
M. Gámez ${ }^{a}$ I. López ${ }^{a}$ J. Garay ${ }^{b}$ Z. Varga ${ }^{c}$ \\ a Department of Statistics and Applied Mathematics, University of Almería, Spain \\ ${ }^{\mathrm{b}}$ Ecological Modelling Research Group of the Hungarian Academy of Science and \\ Department of Plant Taxonomy and Ecology, Eötvös Lorand University, Budapest, \\ Hungary \\ ${ }^{\mathrm{c}}$ Institute of Mathematics and Informatics, Szent István University, Gödölló, \\ Hungary
}

\begin{abstract}
The effect of radiation on a cell population is described by a two-dimensional nonlinear system of differential equations. If the radiation rate is not too high, the system is known to have an asymptotically stable equilibrium. First, for the monitoring of this effect, the concept of observability is applied. For the case when the total number of cells is observed, without distinction between healthy and affected cells, a so-called observer system is constructed, which, at least near the equilibrium state, makes it possible to recover the dynamics of both the healthy and the affected cells, from the observation of the total number of cells without distinction.

Results of simulations with illustrative data are also presented. If we want to control the system into a required new equilibrium state, and maintain this new equilibrium by a constant control, a technique of theory of optimal control can be applied to construct a feedback control system.
\end{abstract}

Key words: population dynamics, observability, observer system, cell radiation.

Email addresses: mgamez@ual.es corresponding author, tel: (34)950015667, fax:(34)950015167 (M. Gámez), milopez@ual .es (I. López), garayj@ludens.elte.hu (J. Garay), Varga.Zoltan@gek.szie.hu (Z. Varga). 


\section{Introduction}

The effect of radiation on a cell population has been modeled and studied by a great number of authors, see e.g. Schöllnberger et al. (1999), Sachs et al. (2001), Belostotski and Freedman (2005). Our investigations will be based on the model considered in Freedman and Pinho (2008), where a two-dimensional nonlinear system of ordinary differential equations describes the respective dynamics of the population of the healthy cells and those affected by radiation, and the interaction between them. In this model it is supposed that the growth of the healthy cells is logistic.

In Section 2 from Freedman and Pinho (2008), we recall the description of the model and some stability results. Section 3 is devoted to the problem of observation. First, in terms of the model parameters, we obtain a sufficient condition for local observability near equilibrium. Then we also prove a theorem concerning the construction of observer systems for different observation situations. The observer system makes it possible for us to asymptotically estimate unknown (i.e. not observed) components of the original system. Illustrative simulation results are also provided. For earlier applications of the concept of observability and observer systems, see e.g. Varga et al. (2003), López et al. (2007), Varga (2008) and Gámez et al. (2008). In Section 4 with illustrative data we will show how the number of healthy cells can be steered to a desired level, applying an appropriate feedback control. Finally, in Section 5 , the methodology and the results are discussed.

\section{Description of the model and preliminaries}

We consider a dynamic model describing the interaction between the populations of healthy and radiated cells of an organ under the effect of a constant radiation. Let $x_{1}(t)$ be the number of healthy cells, and $x_{2}(t)$ the number of radiated cells at time $t$. For this situation, from Freedman and Pinho (2008) we recall the following model:

$$
\begin{aligned}
& \dot{x_{1}}=r x_{1}\left(1-\frac{x_{1}}{K_{0}}\right)-\Delta x_{1}+p x_{2} \\
& \dot{x_{2}}=\Delta x_{1}-p x_{2}-\delta x_{2} .
\end{aligned}
$$


In this systems it is supposed that the growth of the healthy cells is logistic as in Sachs et al.(1992) and Andronov et al. (1973). The number of radiated cells with broken chromosomes are represented by $\Delta x_{1}$, where $\Delta>0$ is the radiation rate determined by the corresponding protocol, $p>0$ is the rate at which the radiated cells recombine into healthy cells, $\delta>0$ is the washout rate of radiated cells, and finally, $r>0$ is the Malthus parameter and $K_{0}>0$ the carrying capacity for the healthy cell population.

Freedman and Pinho (2008) proved that the system leaves the nonnegative orthant invariant. Moreover, they proved that system (1) has always a trivial equilibrium $(0,0)$, and a "mathematical" equilibrium $x^{*}=\left(x_{1}^{*}, x_{2}^{*}\right)$, with

$$
x_{1}^{*}=\frac{K_{0}[r(p+\delta)-\Delta \delta]}{r(p+\delta)}, \quad x_{2}^{*}=\frac{K_{0} \Delta[r(p+\delta)-\Delta \delta]}{r(p+\delta)^{2}} .
$$

Obviously $x^{*}>0$ if and only if

$$
\Delta<\frac{r(p+\delta)}{\delta} .
$$

Below from Freedman and Pinho (2008) we recall three statements. Later on, we will use the Jacobian of the right-hand side of (1) calculated at equilibrium $x^{*}$ :

$$
A=\left(\begin{array}{cc}
r-\frac{2 r x_{1}^{*}}{K_{0}}-\Delta & p \\
\Delta & -p-\delta
\end{array}\right)
$$

\section{Theorem 2.1}

a) $x^{*}>0$ if and only if equilibrium $(0,0)$ is unstable.

b) If $x^{*}>0$, it is globally asymptotically stable, with respect to $\mathbb{R}_{+}^{2} \backslash\{(0,0)\}$.

c) System (1) persists (uniformly) if and only if we have (3). Otherwise the cell population becomes extinct.

\section{Observability analysis of the model}

First we recall some known concepts and results concerning observation systems, to be used in this paper.

Given $m, n$ positive integers, we suppose that the following functions

$$
f: \mathbb{R}^{n} \rightarrow \mathbb{R}^{n}, \quad h: \mathbb{R}^{n} \rightarrow \mathbb{R}^{m}
$$


are continuously differentiable and for some $x^{*} \in \mathbb{R}^{n}$ we have that $f\left(x^{*}\right)=0$ and $h\left(x^{*}\right)=0$.

We consider the following observation system

$$
\begin{aligned}
\dot{x} & =f(x) \\
y & =h(x)
\end{aligned}
$$

where $h$ is the observation function.

Definition 3.1 Observation system (5)-(6) is called locally observable near the equilibrium $x^{*}$ over a given time interval $[0, T]$, if there exists $\epsilon>0$, such that for any two different solutions $x$ and $\bar{x}$ of system (5) with $\left|x(t)-x^{*}\right|<\epsilon$ and $\left|\bar{x}(t)-x^{*}\right|<\epsilon(t \in[0, T])$, the observed functions $h \circ x$ and $h \circ \bar{x}$ are different. (o denotes the composition of functions. For brevity, the reference to $[0, T]$ will be omitted).

For the formulation of a sufficient condition for local observability consider the linearization of the observation system (5)-(6) around $x^{*}$, consisting in the calculation of the Jacobians

$$
A:=f^{\prime}\left(x^{*}\right) \quad \text { and } \quad C:=h^{\prime}\left(x^{*}\right) .
$$

Theorem 3.2 (Lee and Markus, 1971). Suppose that

$$
\operatorname{rank}\left[C|C A| C A^{2}|\ldots| C A^{n-1}\right]^{T}=n .
$$

Then the observation system (5)-(6) is locally observable near the equilibrium $x^{*}$.

Definition 3.3 A matrix $A \in \mathbb{R}^{n \times n}$ will be called stable, if all its eigenvalues have negative real parts.

We remind then how it is possible to construct the observer of a system. Now, the construction of an observer system will be based on Sundarapandian (2002).

Definition 3.4 Given a continuously differential function $G: \mathbb{R}^{n} \times \mathbb{R}^{n} \rightarrow \mathbb{R}^{n}$, dynamical system described by

$$
\dot{z}=G(z, y)
$$


is called a local asymptotic (respectively, exponential) observer for observation system (5)-(6), if the composite system (5)-(6),(8) satisfies the following two requirements.

i) If $x(0)=z(0)$, then $x(t)=z(t)$, for all $t \geq 0$.

ii) There exists a neighborhood $V$ of the equilibrium $x^{*}$ of $\mathbb{R}^{n}$ such that for all $x(0), z(0) \in V$, the estimation error $z(t)-x(t)$ decays asymptotically (respectively, exponentially) to zero.

Theorem 3.5 (Sundarapandian, 2002). Suppose that $x^{*}$ is a Lyapunov stable equilibrium of system (5), and that there exists a matrix $K$ such that matrix $A-K C$ is stable, where $A=f^{\prime}\left(x^{*}\right)$ and $C=h^{\prime}\left(x^{*}\right)$. Then dynamic system defined by

$$
\dot{z}=f(z)+K[y-h(z)]
$$

is a local exponential observer for observation system (5)-(6).

For the application of the above construction we will suppose $x^{*}>0$.

Case 1. We assume that the total number of cells is observed, i.e. the observation function is

$$
h\left(x_{1}, x_{2}\right):=x_{1}+x_{2}-x_{1}^{*}-x_{2}^{*} .
$$

Then for the observation system (1)-(10) we calculate the linearization around the equilibrium $x^{*}$ :

$$
A=\left(\begin{array}{cc}
-r+\frac{2 \Delta \delta}{p+\delta}-\Delta & p \\
\Delta & -p-\delta
\end{array}\right), \quad C=\left(\begin{array}{ll}
1 & 1
\end{array}\right)
$$

It is easy to prove that $\operatorname{rank}[C \mid C A]^{T}=2$ if and only if $\Delta \neq \frac{(r-\delta)(p+\delta)}{2 \delta}$. In this case, by Theorem 3.2 system (1)-(10) is locally observable.

Case 2. Now we present another result on observability, where only the radiated cells are observed. Then the observation function is given by,

$$
h\left(x_{1}, x_{2}\right):=x_{2}-x_{2}^{*} .
$$

Hence, for the linearization of the observation system (1)-(11) we have

$$
A=\left(\begin{array}{cc}
-r+\frac{2 \Delta \delta}{p+\delta}-\Delta & p \\
\Delta & -p-\delta
\end{array}\right), \quad C=\left(\begin{array}{ll}
0 & 1
\end{array}\right) .
$$


Now we have $\operatorname{rank}[C \mid C A]^{T}=2$ without any further condition. Therefore, again by Theorem 3.2 system (1)-(11) is locally observable.

For the construction of observer systems corresponding to Cases 1 and 2, we will prove the following

Theorem 3.6 If for $k_{1}, k_{2} \in \mathbb{R}$ inequalities

(i) $k_{2}>\max \{r, \delta\}$

(ii) $k_{1}>p+2\left(\delta+\frac{r k_{2}}{\Delta}\right)$

hold, then with the corresponding choice of $h$, for systems (1)-(10) and (1)(11), a local observer is given by

$$
\begin{aligned}
& \dot{z_{1}}=r z_{1}\left(1-\frac{z_{1}}{K_{0}}\right)-\Delta z_{1}+p z_{2}+k_{1}[y-h(z)] \\
& \dot{z_{2}}=\Delta z_{1}-p z_{2}-\delta z_{2}+k_{2}[y-h(z)] .
\end{aligned}
$$

Proof. In both cases, denoting $K:=\operatorname{col}\left(k_{1}, k_{2}\right)$ and applying the RouthHurwitz criterion for $n=2$, we have to guarantee that the coefficients of the normed characteristic polynomial of matrix $A-K C$ are positive, i.e. in Cases 1 and 2, the following inequalities should hold:

Case 1.

$$
\begin{array}{r}
r-\frac{2 \Delta \delta}{p+\delta}+\Delta+p+\delta+k_{1}+k_{2}>0 \\
\left(-r+\frac{2 \Delta \delta}{p+\delta}-\Delta-k_{1}\right)\left(-p-\delta-k_{2}\right)-\left(\Delta-k_{2}\right)\left(p-k_{1}\right)>0
\end{array}
$$

Case 2.

$$
\begin{array}{r}
r-\frac{2 \Delta \delta}{p+\delta}+\Delta+p+\delta+k_{2}>0 \\
\left(-r+\frac{2 \Delta \delta}{p+\delta}-\Delta\right)\left(-p-\delta-k_{2}\right)-\Delta\left(p-k_{1}\right)>0
\end{array}
$$

Now, from (3) we immediately get $-r+\frac{2 \Delta \delta}{p+\delta}<r$, furthermore, (i) and (ii) imply $k_{1}+k_{2}>r$. Hence we easily obtain (12) and (14). Based on this, to see 
(13) it is enough to show that

$$
k_{1}>\frac{(r-\Delta)\left(\delta+k_{2}\right)}{\Delta} .
$$

By $(i)$ we have $r \delta<r k_{2}$, implying

$$
(r-\Delta)\left(\delta+k_{2}\right)<2 r k_{2}+2 \Delta \delta+\Delta p \Longrightarrow \frac{(r-\Delta)\left(\delta+k_{2}\right)}{\Delta}<p+2\left(\delta+\frac{r k_{2}}{\Delta}\right) .
$$

Hence, applying (ii) we obtain (16).

Finally, to see (15), it is enough to show

$$
\frac{2 \Delta \delta}{p+\delta}\left(-p-\delta-k_{2}\right)-\Delta\left(p-k_{1}\right)>0
$$

For the latter it is sufficient to prove inequality

$$
k_{1}>2 \delta+\frac{2 \delta k_{2}}{p+\delta}+p
$$

which is implied by $(i i)$, since from (3) we have

$$
p+2\left(\delta+\frac{r k_{2}}{\Delta}\right)>2 \delta+\frac{2 \delta k_{2}}{p+\delta}+p
$$

Now, the application of Theorem 3.5 concludes the proof.

Example. For an illustration set $\Delta:=2, r:=2.1, K_{0}:=100, p:=1, \delta:=$ 0.1 In this case $x^{*}>0$ and is globally stable. Moreover, we easily get the corresponding rank condition for local observability in both Cases 1 and 2 . On the other hand, for these parameter values, the conditions $(i)$ and $(i i)$ of Theorem 3.6 are satisfied:

$$
k_{2}>2.1 \quad, \quad k_{1}>1+2\left(0.1+1.05 k_{2}\right) .
$$

Therefore, with $K:=\operatorname{col}(150,50)$ we obtain the following observer system:

$$
\begin{aligned}
& \dot{z}_{1}=2.1 z_{1}\left(1-\frac{z_{1}}{100}\right)-2 z_{1}+z_{2}+150(y-h(z)) \\
& \dot{z}_{2}=2 z_{1}-z_{2}-0.1 z_{2}+50(y-h(z)) .
\end{aligned}
$$

Let us consider Case 1, and solve systems (1) and (17) with the respective initial conditions $x(0)=(100,0)$ and $z(0)=(150,50)$, with observation function (10). 
In Figure 1 we can see that the observer system (17) asymptotically recovers the solution of the original system.

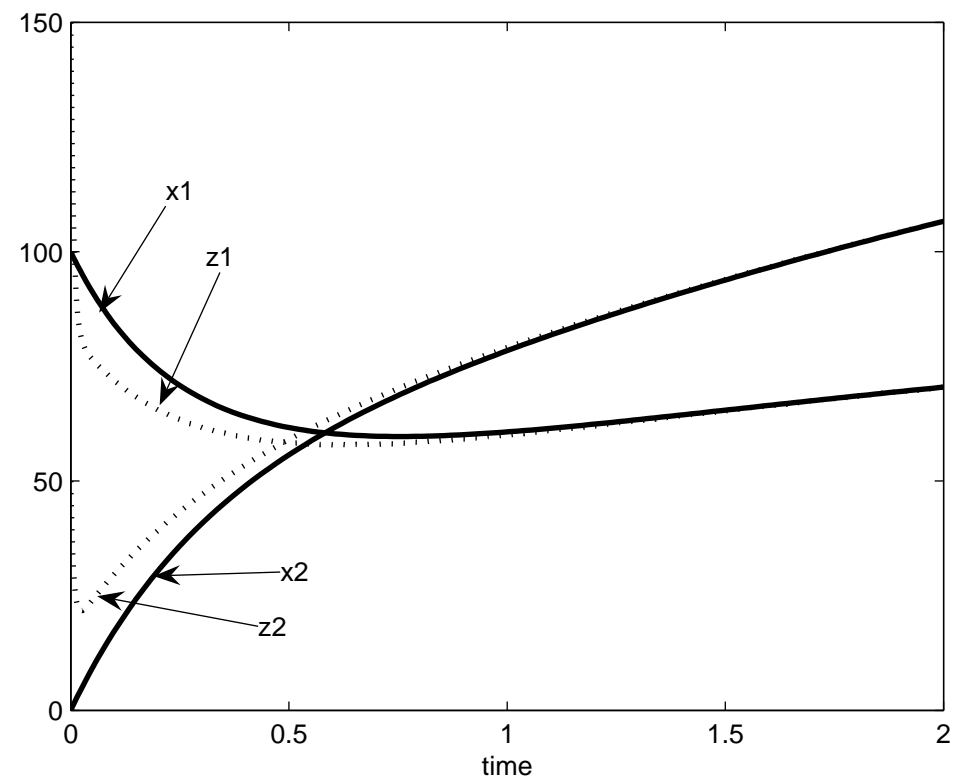

Fig. 1. Solutions of (1) and (17) with initial conditions $x(0)=(100,0)$ and $z(0)=(150,50)$

Similarly, if we consider now Case 2, i.e. observation function (11), and solve system $(17)$ with $z(0)=(150,50)$, solution $z$ approaches the solution of the original system $(1)$ with the initial condition $x(0)=(100,0)$.(See Figure 2.)

\section{Linear feedback control for the radiation}

For $n, r \in \mathbb{N}, L \in \mathbb{R}^{n \times n}, B \in \mathbb{R}^{n \times r}$, and continuously differentiable function $g: \mathbb{R}^{n} \rightarrow \mathbb{R}^{n}$, consider the control system

$$
\dot{x}=L x+g(x)+B U,
$$

where $U$ is a continuous control function. Assume that to a constant control $u^{*} \in \mathbb{R}^{r}$, there corresponds an equilibrium state $x^{*}$, i.e.,

$$
L x^{*}+g\left(x^{*}\right)+B u^{*}=0 .
$$




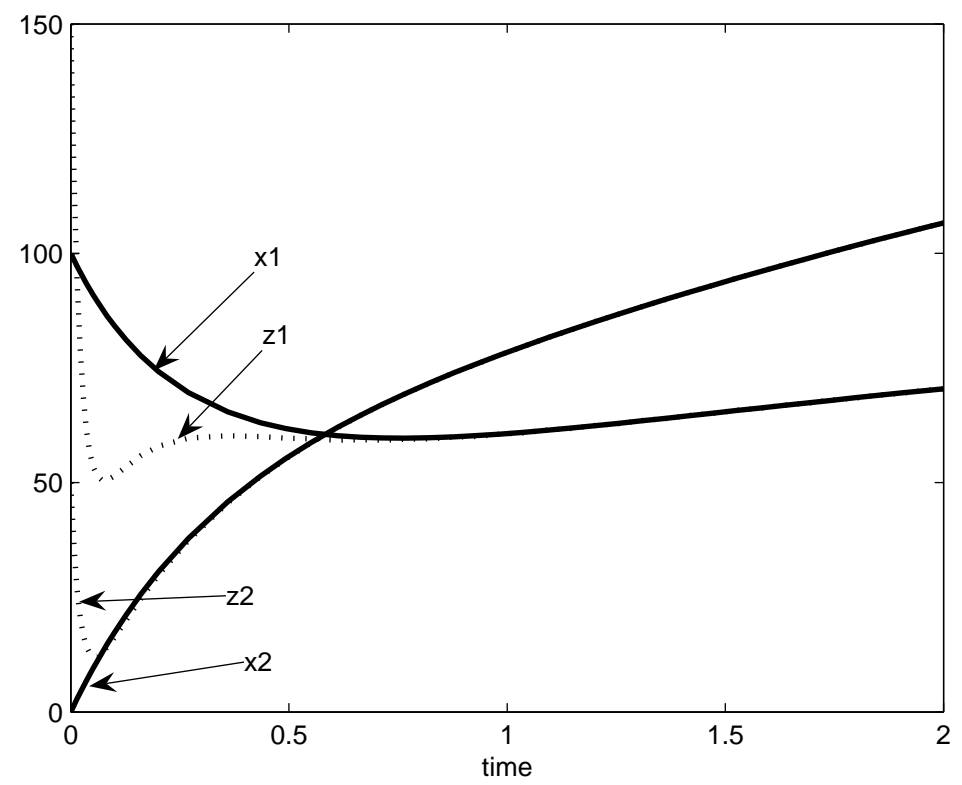

Fig. 2. Solutions of (1) and (17) with initial conditions $x(0)=(100,0)$ and $z(0)=(150,50)$

Then, from (18) and (19), for the new variables

$$
y:=x-x^{*} \quad ; \quad u:=U-u^{*}
$$

we have

$$
\dot{y}=L y+q(y)+B u, \quad \text { with } \quad q(y):=g\left(y+x^{*}\right)-g\left(x^{*}\right) .
$$

Below a feedback control will be given which asymptotically steers system (20) into the zero equilibrium.

Theorem 4.1 (Rafikov et al. 2008) If there exist matrices $P, Q, R \in \mathbb{R}^{n \times n}$; $P$ positive definite and $Q$ symmetric, such that the function

$$
l(y):=y^{T} Q y-q^{T}(y) P y-y^{T} P q(y) \quad\left(y \in \mathbb{R}^{n}\right)
$$

is positive definite, and $P$ satisfies the equation

$$
P L+L^{T} P-P B R^{-1} B^{T} P+Q=0 .
$$

Then the linear feedback

$$
u(y):=-R^{-1} B^{T} P y \quad\left(y \in \mathbb{R}^{n}\right)
$$


asymptotically steers any initial state $y(0)$ to zero.

Remark 4.2 The statement $\lim _{\infty} y=0$ is obviously equivalent to $\lim _{\infty} x=$ $x^{*}$.

Remark 4.3 According to Rafikov et al. 2008, the feedback control (22) also minimizes the functional

$$
\phi(y):=\int_{0}^{\infty}\left[l(y(t))+u^{T}(y(t)) R u(y(t))\right] d t,
$$

however, we will not use this statement.

Corollary 4.4 Using the notation of the previous theorem, let us suppose that function $l$ is locally positive definite. Then there exists a neighbourhood $V$ of zero in $\mathbb{R}^{n}$ such that for all $x(0) \in V$, for the solution $x$ of system (19) we have $\lim _{\infty} x=x^{*}$.

Proof. The proof of Theorem 4.1 available in Rafikov et al. 2007, is based on the observation that under the conditions of the mentioned theorem, $W(y)=$ $y^{T} P y$ is a Lyapunov function implying (global) asymptotic stability of the zero equilibrium of system (20). It is not hard to see that a similar reasoning implies the following statement. If function $W$ is locally positive definite, then the zero equilibrium of system (20) is locally asymptotically stable. Hence, the statement of the Corollary follows.

Now, we are going to apply the above corollary to system (1) to control cell populations, utilizing the following control system

$$
\begin{aligned}
& \dot{x_{1}}=r x_{1}\left(1-\frac{x_{1}}{K}\right)-\Delta x_{1}+p x_{2} \\
& \dot{x_{2}}=\Delta x_{1}-p x_{2}-\delta x_{2}+U,
\end{aligned}
$$

corresponding to system (18).

Our objective is to find a feedback control that steers the population of healthy cells to a desired level $x_{1}^{*}=x_{1 d}$. The corresponding value $x_{2}^{*}=x_{2 d}$ and $u^{*}$ can be calculated solving the following system of linear equations:

$$
\begin{aligned}
r x_{1}^{*}\left(1-\frac{x_{1}^{*}}{K}\right)-\Delta x_{1}^{*}+p x_{2}^{*} & =0 \\
\Delta x_{1}^{*}-p x_{2}^{*}-\delta x_{2}^{*}+u^{*} & =0 .
\end{aligned}
$$


We note that $u^{*}$ is interpreted as a constant radiation rate that would maintain the desired level $x_{1}^{*}=x_{1 d}$ of healthy cells.

Now, for systems (21) and (22), we can choose

$$
L:=\left(\begin{array}{cc}
r-\Delta & p \\
\Delta & -(p+\delta)
\end{array}\right)
$$

and

$$
h(y):=\left(\begin{array}{c}
-\frac{r}{K}\left(y_{1}^{2}+2 y_{1} x_{1}^{*}\right) \\
0
\end{array}\right) \quad\left(y \in \mathbb{R}^{2}\right) .
$$

If the conditions of Theorem 4.1 are satisfied, the required feedback control can be obtained by (22).

For the parameters considered in previous model simulations $r:=1, \Delta:=$ $5, K:=100, p:=1$, and $\delta:=0.1$, it is easy to prove that system (1) has an asymptotically stable equilibrium, where $x_{1}=54$. Now, we suppose that the objective is to increase the number of healthy cells, for example to a level $x_{1 d}=80$. To this end, from system (25) we calculate $x_{2 d}=344$ and $u^{*}=21.6$. For matrices $L$ and $B$ we have

$$
L=\left[\begin{array}{cc}
-4 & 1 \\
5 & -1.1
\end{array}\right], \quad B=\left[\begin{array}{l}
0 \\
1
\end{array}\right]
$$

Choosing

$$
Q:=\left[\begin{array}{ll}
1 & 0 \\
0 & 1
\end{array}\right], \quad R=[1],
$$

we calculate the matrix Riccati equation (21), and using the function LQR of $M A T L A B^{T M}$ v 7.0, we obtain a solution

$$
P=\left[\begin{array}{ll}
1.2362 & 0.9862 \\
0.9862 & 0.9451
\end{array}\right]
$$


Obviously $P$ and $Q$ are positive definite symmetric matrices. Furthermore, it is easy to verify analytically that $(0,0)$ is a local minimum point for function $l$ (see also Figure 3), and by the Corollary to Theorem 4.1, we have the local asymptotic stability of the zero equilibrium of system (20). Therefore, applying (22), we obtain the required feedback control

$$
u=-0.9862 y_{1}-0.9451 y_{2}
$$

Hence, from inequalities $x=x^{*}+y$ and $U=u^{*}+u$, we can calculate the closed loop control system

$$
\begin{aligned}
& \dot{x_{1}}=x_{1}\left(1-\frac{x_{1}}{100}\right)-5 x_{1}+x_{2} \\
& \dot{x_{2}}=4.0138 x_{1}-2.045 x_{2}+425.576 .
\end{aligned}
$$

Figure 4 shows how the second coordinate of the solution of the controlled system asymptotically reaches the desired value $x_{1 d}=80$.

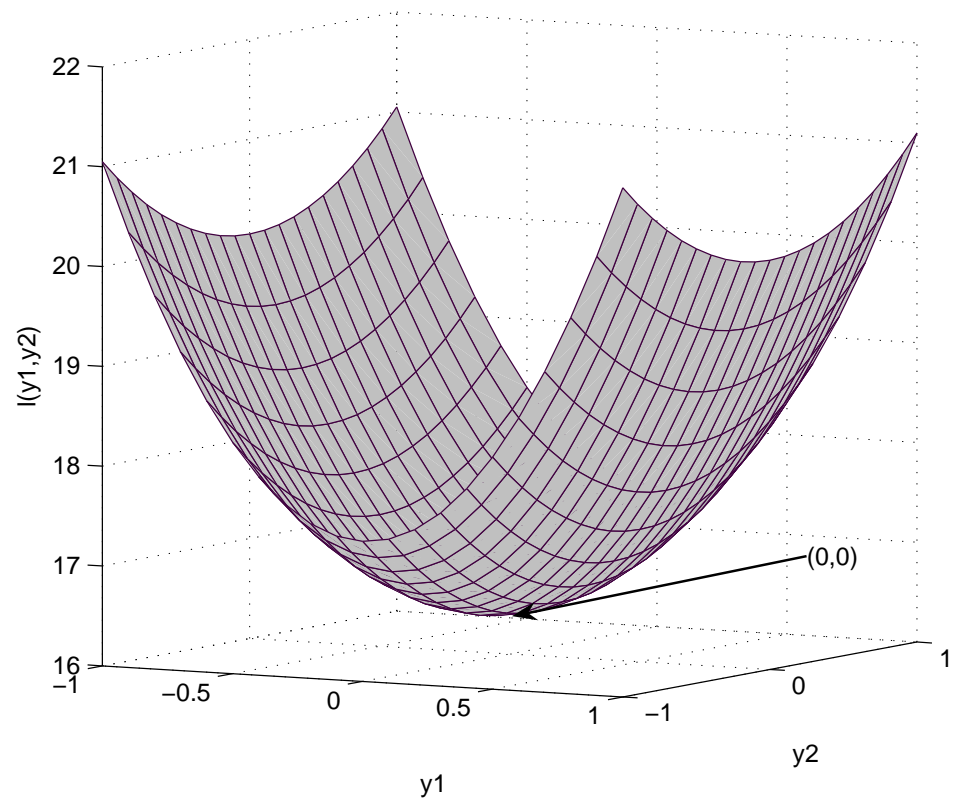

Fig. 3. Function $l(y)$ near equilibrium $(0,0)$. 


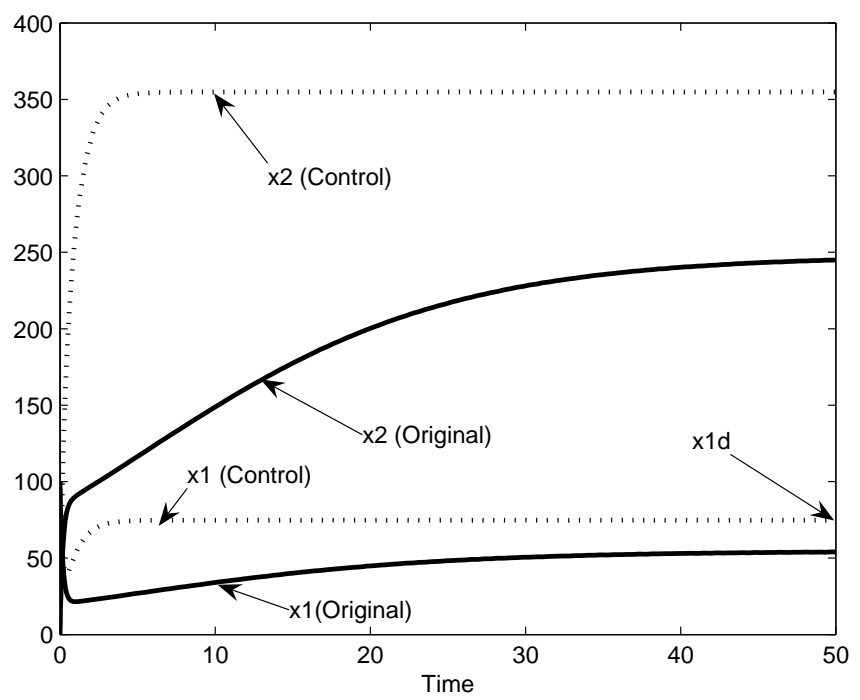

Fig. 4. Solutions of (1) and (27) with the same initial value $x(0)=(100,0)$

In Figure 5 we show the evolution of function $U(t)$ in the controlled system.

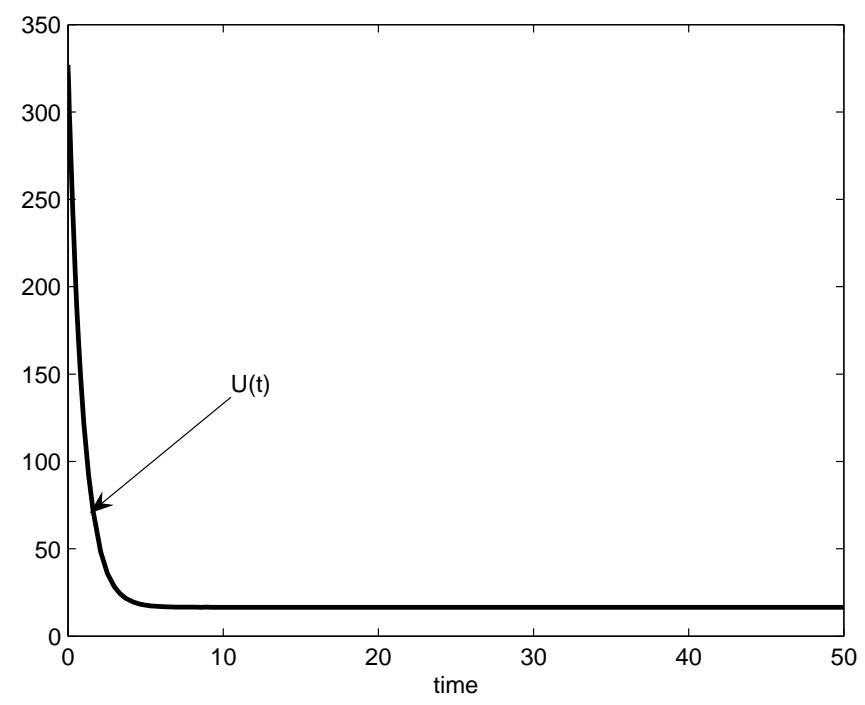

Fig. 5. Function $U(t)$ in the controlled system (27)

As seen from Figure 5, function $U(t)$ is always positive, which corresponds to its physical interpretation as radiation intensity. 


\section{Discussion}

Using known results on the existence of an asymptotically stable equilibrium in a cell radiation model, in the paper sufficient conditions have been obtained for the local observability and for the observer design, corresponding to different observation situations. As a result, on the one hand, we can estimate both the population of healthy cells and those affected by the radiation, provided the total number of cells is observed. Similarly, from the observation of the radiated cells we can recover the number of healthy cells. In both cases, by an appropriate choice of two auxiliary parameters, we achieve a quite quick convergence of the asymptotic estimation.

We have also shown, how a desired number of healthy cells can be produced, using a feedback control. We note that in the resulting closed-loop system the system practically reaches the equilibrium much more quickly than in the original system.

Finally, the methodology we offered can be easily extended to other cell radiation models, too.

\section{Acknowledgements}

This publication has been realized with the support of the Hungarian Scientific Research Fund (OTKA 62000 and 68187). The authors wish to thank the Ministry of Education and Science of Spain for the project TIN2007-67418C03-02, which has partially supported this work.

\section{References}

Andronov, A.A., Leontovich, E.A., Gordon, I.I. and Maier, A.G., 1973. Qualitative Theory of Second-Order Dynamic Systems. Halstead Press, New York.

Belostotski, G. and Freedman, H.I., 2005. A control theory model for cancer treatment by radiotherapy. Int. Jour. Pure Appl. Math. 25, 447-480. 
Freedman, H.I. and Pinho, S.T.R., 2008. Persistence and extinction in a mathematical model of cell populations affected by radiation. Periodica Mathematica Hungarica, 56 (1), 25-35.

Gámez, M.; López, I. and Molnár, S., 2008. Monitoring environmental change in an ecosystem. Biosystems, 93, 211-217.

Lee, E.B. and Markus, L., 1971. Foundations of Optimal Control Theory. New York-London-Sydney : Wiley.

López, I.; Gámez, M.; Garay, J. and Varga, Z., 2007. Monitoring in a LotkaVolterra model. Biosystems, 87, 68-74.

Rafikov, M., Balthazar, J.M., Bremen, H.F. von., 2008. Mathamatical Modelling and Control of Population Systems: Applications in Biological Pest Control. Appl. Math. Comput., 200 (2), 557-573

Sachs, Chen, P.L., R.K., Hahnfeldt, P. and Hlatky, L.R., 1992. DNA danage caused by ionizing radiation. Math. Biosci. 112, 271-303.

Sachs, R.K., Hlatky, L.R. and Hahnfeldt, P., 2001. Simple ODE models of tumor growth and anti-angiogenic or radiation treatment. Math. Comput. Model 33, 1297-1305.

Schöllnberger, H., Kotecki, M., Crawford-Brown, D., Hofmann, W. and Echl, E., 1999. Adaptive response plateaus for initiation in a statevector model of carcinogenesis. Int. Jour. Radiation Biol., 75, 351-364.

Sundarapandian, V., 2002. Local observer design for nonlinear systems. Mathematical and computer modelling 35, 25-36.

Varga, Z., 2008. Applications of mathematical systems theory in population biology. Periodica Mathematica Hungarica, 51 (1), 157-168.

Varga, Z., Scarelli, A. and Shamandy, A., 2003. State monitoring of a population system in changing environment. Community Ecology 4 (1), 73-78. 